\title{
Sinoatrial conduction abnormalities - an underestimated cardiac complication in women with systemic sclerosis
}

\author{
Elżbieta Kramarz'1, Lidia Rudnicka², Zbigniew Samochocki²
}

${ }^{1}$ Internal Medicine and Cardiology Department, Military Institute of Medicine, Warsaw, Poland ${ }^{2}$ Department of Dermatology, Medical University of Warsaw, Warsaw, Poland

Adv Dermatol Allergol 2021; XXXVIII (2): 269-273

DOI: https://doi.org/10.5114/ada.2021.106203

\begin{abstract}
Introduction: It has been claimed that patients with systemic sclerosis (SSc) have an increased risk of developing cardiac arrhythmias and atrioventricular conduction disorders, but it is unknown whether SSc may be a cause of sinoatrial conduction abnormalities.

Aim: To establish the incidence of sinoatrial conduction abnormalities in patients with SSc and verify the relationship of these disorders with various clinical descriptors of SSc.

Material and methods: Forty women with systemic sclerosis of varying duration and severity underwent 24-hour ambulatory ECG monitoring. The occurrence of type I second-degree sinoatrial block (SA-block) and calculation of sinoatrial conduction time (SACT) were evaluated to establish the incidence of sinoatrial conduction abnormalities. The measurements of SACT were obtained using spontaneous atrial premature beats. The effect of various clinical descriptors on sinoatrial conduction abnormalities was assessed.

Results: The mean \pm SD SACT for the 40 patients was $150 \pm 15 \mathrm{~ms}$. Prolonged (> $150 \mathrm{~ms}$ ) SACT was found in 20 patients. In 14 (35\%) patients SA-block occurred during ambulatory ECG monitoring. The discriminant analysis identified the severity of SSc cutaneous manifestation as an independent marker for developing SA-block $(p<0.005)$ and SACT prolongation $(p<0.0002)$.

Conclusions: Patients with SSc are at an increased risk of developing type I second-degree sinoatrial block and prolonged sinoatrial conduction time. The occurrence of these abnormalities is related to the severity of skin involvement. Therefore, cardiological diagnosis using 24-hour ambulatory ECG in this group of patients should be focused also on this type of disorders. Prospective, controlled studies are needed to assess their prognostic role.
\end{abstract}

Key words: heart disease, systemic sclerosis, sinoatrial conduction abnormalities.

\section{Introduction}

Abnormalities in the circulatory system in patients with systemic sclerosis (SSc) have been repeatedly reported in the medical literature [1-5]. Systemic sclerosis is characterized by a disproportion between mild clinical symptoms and progressive inconspicuous internal organ involvement [6]. It has been shown that various types of ECG abnormalities are present in 25-98\% of patients with SSc [7, 8]. ECG abnormalities are various and non-specific. The most frequent changes include low voltage of QRS complex, ST segment and T wave abnormalities, right and left bundle branch block, features of atrial and/or ventricular hypertrophy, supraventricular or ventricular arrhythmias. Holter monitoring allows to detect various types of arrhythmias and conduction disorders in the majority of
SSc patients. Atrioventricular (AV) conduction disorders such as first-, second- and third-degree atrioventricular block, intraventricular conduction perturbations, right or left bundle branch block were often described in this group of patients [8]. The degree of cardiac involvement is one of the main negative predictors of clinical course and prognosis in SSc patients. Bryan et al. [9] noted that abnormal resting ECG is one of the most important risk factors for death. Heart involvement in the course of SSc is responsible for approximately $40 \%$ of deaths and requires specific clinical management [7]. Even minor ECG abnormalities, which could indicate the activity and progression of cardiac fibrosis, may have a significant prognostic value in SSc patients. There are many studies on the occurrence of supraventricular and ventricular arrhythmias,

Address for correspondence: Prof. Lidia Rudnicka MD, PhD, Department of Dermatology, Medical University of Warsaw, 82 A Koszykowa St, 02-008 Warsaw, Poland, phone/fax: +48 2250213 24, e-mail: lidiarudnicka@gmail.com

Received: 6.10.2019, accepted: 26.10.2019. 
atrioventricular and intraventricular (right or left bundle branch block) conduction abnormalities in the course of systemic sclerosis. However, there are no studies on sinoatrial conduction abnormalities in SSc patients. It should be noted that it is difficult to diagnose these cardiological abnormalities on the basis of a standard ECG. ECG Holter monitoring enables the diagnosis of both first-degree sinoatrial block and type I second-degree sinoatrial block. These arrhythmias are clinically important because they may indicate-significant sinus node dysfunction that could even require constant cardiac pacing.

\section{Aim}

The aim of this study was to assess the occurrence of sinoatrial conduction abnormalities in the group of patients with SSc by performing a 24-hour ECG monitoring and to find the best clinical predictors that discriminate between the patients with and without sinoatrial disorders.

\section{Material and methods}

The study population consisted of 40 adult women with documented systemic sclerosis in whom 24-hour ambulatory ECG monitoring had shown the occurrence of atrial premature beats that fulfilled the mentioned criteria. Characteristics of the examined group are shown in Table 1.

Systemic sclerosis was diagnosed according to the American College of Rheumatology criteria [10]. The project was approved by the local ethics committee. All participants gave their written consent prior to the study.

\section{Clinical and laboratory examinations}

All patients underwent physical examination and anamnesis. Severity of cutaneous manifestation was rated using Rodnan skin score [11].
Indirect immunofluorescence was used to screen all patients for serological markers: anti-centromere antibodies (ACA) and anti-topoisomerase (Scl-70), which were confirmed by immunoblot assay, taking the titre of $1: 160$ as a positive result.

\section{4-hour ambulatory ECGs}

24-hour ambulatory ECGs were obtained using threechannel recorders with bipolar leads: CM5, CS2 and IS. Measurements of sinoatrial conduction time were performed on the first fragment of ambulatory ECG with premature atrial beat that fulfilled the following criteria: 1) the premature atrial depolarization with positive $P$ wave in the lead CM5 occurred during normal daily activities at a sinus rate of 60-90 bpm, 2) the differences in the cycle length between three consecutive PP intervals preceding premature atrial beats were lower than $20 \mathrm{~ms}$, 3 ) the coupling interval of atrial premature depolarization was longer than $50 \%$ of the preceding sinus cycle length, 4) the sum of the coupling interval and the postextrasystolic pause was shorter than the sum of two spontaneous sinus cycles.

The following time intervals (Figure 1) were estimated: (P1-P1) - the basic cycle length, i.e., the interval between the last two sinus $P$ waves before the premature atrial beat; and (P2-P3) - the return cycle length, i.e., the interval between premature atrial beat and the next sinus $P$ wave. Sinoatrial conduction time (SACT) was calculated according to the formula: $\mathrm{SACT}=(\mathrm{P} 2-\mathrm{P} 3-\mathrm{P} 1-\mathrm{P} 1) / 2$. According to the results of the previous studies, the values of sinoatrial conduction time greater than $150 \mathrm{~ms}$ were considered prolonged [12].

Type I second-degree sinoatrial block (SA-block) was recognized when the sequence of gradually shorter PP interval was followed by a longer PP interval which was less than twice the length of the preceding PP interval (Figure 1).

Table 1. Clinical characteristics of forty women with systemic sclerosis

\begin{tabular}{lccccccccc}
\hline Variable & Min. & Max. & Mean & SD & Median & $\begin{array}{c}\text { First } \\
\text { quartile }\end{array}$ & $\begin{array}{c}\text { Third } \\
\text { quartile }\end{array}$ & $\begin{array}{c}\text { Shapiro-Wilk } \\
p \text {-value }\end{array}$ & $\begin{array}{c}\text { Normal } \\
\text { distribution }\end{array}$ \\
\hline Age [years] & 29 & 80 & 54.8 & 12.9 & 57 & 42 & 61.5 & 0.3625 & Yes \\
\hline Disease duration [years] & 1 & 43 & 8.9 & 8.3 & 7 & 2.5 & 12 & 0.0000 & No \\
\hline Rodnan score [points] & 1 & 23 & 5.6 & 5.8 & 5 & 2 & 6 & $<0.0001$ \\
\hline
\end{tabular}

A

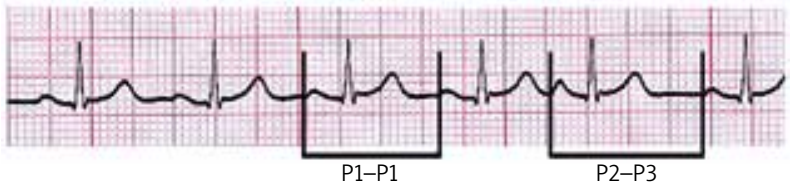

B

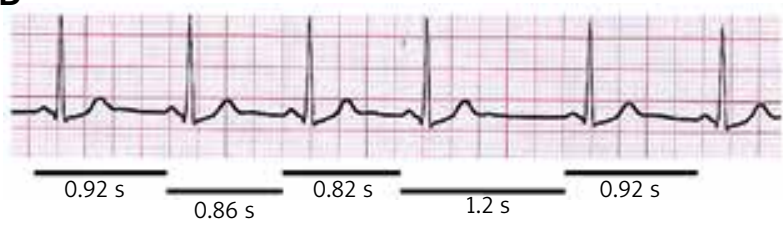

Figure 1. This pattern represents P-P interval before and after atrial premature beat used for the calculation of sinoatrial conduction time (A) and variability of P-P intervals characteristic for type I second-degree sinoatrial block (B) 
Table 2. Univariate relation between evaluated variables and the occurrence of prolonged SACT in women with SSC

\begin{tabular}{lcccc}
\hline Variables & $\begin{array}{c}\text { SACT }>150 \mathrm{~ms} \\
n(\%)\end{array}$ & $\begin{array}{c}\mathrm{SACT} \leq 150 \mathrm{~ms} \\
n(\%)\end{array}$ & $\chi^{2}$ & $P$-value \\
\hline Age $>50$ years & $17(42.5)$ & $7(17.5)$ & 6.08 & 0.0137 \\
\hline Duration of disease $\geq 7$ years & $20(50.0)$ & $9(22.5)$ & 15.17 & 0.0001 \\
\hline Rodnan score $>5$ points & $19(47.5)$ & $5(12.5)$ & 20.42 & 0.0000 \\
\hline Positive Scl-70 test & $12(30.0)$ & $5(12.5)$ & 5.01 & 0.0252 \\
\hline Positive ACA test & $12(30.0)$ & $10(25.0)$ & 0.40 & 0.5250 \\
\hline
\end{tabular}

ACA - anti-centromere antibodies, Scl-70 - anti-topoisomerase antibodies, Rodnan score-a point-score system to estimate sclerosis severity.

Table 3. Discriminant analysis of evaluated predictors of prolonged SACT in women with SSC

\begin{tabular}{lcc}
\hline Predictors & Wilks' $\boldsymbol{\lambda}$ & $\boldsymbol{P}$-value \\
\hline Age $>50$ years & 0.4102 & 0.2104 \\
\hline Duration of disease $>7$ years & 0.3983 & 0.4552 \\
\hline Rodnan score $>5$ points & 0.6944 & 0.0000 \\
\hline Positive Scl-70 test & 0.4556 & 0.0526 \\
\hline Abbreviations as in Table 2. & &
\end{tabular}

Table 5. The discriminant analysis of evaluated predictors of type I second-degree sinoatrial block

\begin{tabular}{lcc}
\hline Predictors & Wilks' $\lambda$ & $P$-value \\
\hline Duration of disease $>7$ years & 0.7577 & 0.2118 \\
\hline Rodnan score $>5$ points & 0.9190 & 0.0033 \\
\hline Abbreviations as in Table 2. & &
\end{tabular}

\section{Statistical analysis}

Continuous data were expressed as mean \pm SD and median. Discrete and dichotomized variables were presented as a percentage of prevalence and analysed using $\chi^{2}$ test. To identify normal distribution, the Shapiro-Wilk normality test was used. The discriminant analysis was used to find the best predictors that discriminate between patients with and without sinoatrial conduction abnormalities. In this analysis, dichotomized descriptors were represented as a value of 1 if present. Differences were regarded as significant at $p<0.05$ level. Complete Statistical System package (Microsoft Corporation, USA) was used for statistical calculations.

\section{Results}

Sinoatrial conduction time (SACT) varied from $130 \mathrm{~ms}$ to $179 \mathrm{~ms}$, and the mean value was $150 \mathrm{~ms}$. First-degree sinoatrial block was diagnosed in 20 (50\%) patients. Mobitz type I second-degree sinoatrial block was reported in 14 (35\%) patients. ACA antibodies were found in 22 (55\%) patients, Sclero 70 antibodies were present in 17 (43\%) patients. Raynaud phenomenon was observed in all patients.
Table 4. Univariate relation between evaluated variables and the occurrence of type II second-degree sinoatrial block (SA-block) in women with SSc

\begin{tabular}{lcccc}
\hline Variables & $\begin{array}{c}\text { SA-block } \\
n(\%)\end{array}$ & $\begin{array}{c}\text { No SA-block } \\
n(\%)\end{array}$ & $\chi^{2}$ & $P$-value \\
\hline Age $>50$ years & $11(27.5)$ & $14(35.0)$ & 2.37 & 0.1234 \\
\hline $\begin{array}{l}\text { Duration of disease } \\
>7 \text { years }\end{array}$ & $13(32.5)$ & $16(40.0)$ & 4.48 & 0.0344 \\
\hline $\begin{array}{l}\text { Rodnan score } \\
>5 \text { points }\end{array}$ & $13(32.5)$ & $11(27.5)$ & 9.69 & 0.0019 \\
\hline Positive Scl-70 test & $8(20.0)$ & $9(22.5)$ & 1.89 & 0.1692 \\
\hline Positive ACA test & $10(25.0)$ & $12(30.0)$ & 2.35 & 0.1254 \\
\hline
\end{tabular}

Abbreviations as in Table 2.

There was a statistically significant relationship between SACT and the age, level of skin involvement, disease duration and the presence of anti-Scl-70 antibodies (Table 2).

The discriminatory analysis showed that only the level of skin involvement expressed as the Rodnan score showed a statistically significant correlation with SACT (Table 3).

There was a significant correlation between the occurrence of type I second-degree sinoatrial block in a 24-hour ECG monitoring and both disease duration and Rodnan score value (Table 4).

The discriminatory analysis showed that the level of skin involvement based on the Rodnan score is the strongest predictor for the occurrence of type I second-degree sinoatrial block over a 24-hour period (Table 5).

First-degree atrioventricular block was found in 22 patients, 12 of which had first-degree sinoatrial block. Fifteen patients had second-degree atrioventricular block, 9 of which had type I second-degree sinoatrial block.

\section{Discussion}

Systemic sclerosis is a chronic autoimmune connective tissue disease that develops mainly in women between the $3^{\text {rd }}$ and $5^{\text {th }}$ decade of life. Based on the extent of skin involvement and the type of immune disorders, 
the disease can be classified into two subsets: limited and diffuse SSc [13]. In both clinical subsets sclerosis may involve various internal organs, including the heart [14]. Structural and functional disorders of the blood vessels and endothelial damage cause Raynaud's phenomenon which may precede skin involvement [15].

Cardiac abnormalities in SSc are caused by myocarditis, progressive myocardial fibrosis and inflammation of small vessels with impaired coronary microcirculation [16]. Focal lesions have been found in the atrioventricular node and proximal bundle branches. The relationship between fibrosis of the atrioventricular node and first-degree atrioventricular block has also been demonstrated [8]. Moreover, atrioventricular conduction abnormalities and bundle branch blocks have often been reported in SSc patients. The measurement of sinoatrial conduction time is useful in the diagnosis of sinus node dysfunction in SSc patients. A non-invasive method of calculating SACT allows to assess the incidence of these disorders in this group of patients.

A correlation between sinoatrial conduction time calculated using the Holter method and the invasive method has been reported [17]. The clinical value of SACT, calculated using the Holter method, was confirmed in patients with syncope of unexplained origin [18]. The value of the sinoatrial conduction time determined with the help of the Holter monitor was also confirmed by ISHNE in 2017 [19].

During Holter monitoring, atrial arrhythmias occur in $42 \%$ to $92 \%$ of healthy individuals [20]. In SSc patients, the occurrence of atrial premature beats is more frequent than in healthy subjects [1, 2]. High incidence of atrial premature beats allows to calculate sinoatrial conduction time using Holter monitoring in the majority of SSc patients. In the present study, Holter monitoring was used to calculate sinoatrial conduction time in all subjects. Mean sinoatrial conduction time in SSc patients was $151 \mathrm{~ms}$ during daily activities, and thus it was longer than in healthy individuals. In patients with idiopathic syncopes, sinoatrial conduction time greater than 150 ms during daily activities is a risk factor for type I and type II atrioventricular block and sinus node disease [12]. This study showed a statistically significant relationship between sinoatrial conduction time and the disease duration, the skin involvement and the presence of antiScl-70 antibodies. However, the skin involvement was the best independent predictor of prolonged sinoatrial conduction time. It is worth noticing that prevalence of type I second-degree sinoatrial block in the study group has been much higher than in the general population with arrhythmia. Prevalence of such Mobitz type I second-degree sinoatrial block in patients with paroxysmal arrhythmia population is estimated at 29\% [21].

Skin involvement was the most significant predictor of Mobitz type I second-degree sinoatrial block over a 24-hour period in our group of SSc patients. Extending ECG Holter monitoring to the assessment of sinoatrial conduction abnormalities provides additional information indicating an increased risk for sinus node disease and sudden death in SSc patients.

Systemic sclerosis is a rare disease, thereby limiting the size of our study group. Nevertheless despite the limited cohort size, consistent abnormalities of sinoatrial conduction and significant associations were found.

\section{Conclusions}

The study shows that prolonged sinoatrial conduction time and episodes of sinoatrial block often occur in systemic sclerosis. These arrhythmias may not be detected on a standard ECG in the absence of premature atrial beats. Therefore, evaluation of ECG Holter monitoring in patients with SSc should focus also on this type of sinoatrial conduction abnormalities.

A significant, independent relationship between the severity of skin involvement in SSc and the prolongation of sinoatrial conduction time as well as the presence of sinoatrial block may suggest that the degenerative process with fibrotic changes involves the sinus node and perinodal zone which results in the development of these cardiological disorders in SSc patients. Prospective, controlled studies are needed to assess the prognostic role of prolonged sinoatrial conduction time and sinoatrial block in patients with SSC.

\section{Conflict of interest}

The authors declare no conflict of interest.

\section{References}

1. Vacca A, Meune C, Gordon J, et al. Cardiac arrhythmias and conduction defects in systemic sclerosis. Rheumatology 2014; 53: 1172-7.

2. Komocsi A, Vorobcsuk A, Faludi R, et al. The impact of cardiopulmonary manifestations on the mortality of SSc: a systematic review and meta-analysis of observational studies. Rheumatology 2012; 51: 1027-36.

3. Elhai M, Meune C, Avouac J, et al. Trends in mortality in patients with systemic sclerosis over 40 years: a systemic review and meta-analysis of cohort studies. Rheumatology 2012; 51: 1017-26.

4. Kahan A, Allanore Y. Primary myocardial involvement in systemic sclerosis Rheumatology 2006; 45 Suppl 4: 14-7.

5. Bielous-Wilk A, Poreba M, Staniszewska-Marszałek E, et al. Electrocardiographic evaluation in patients with systemic scleroderma and without clinically evident heart disease. Ann Noninvasive Electrocardiol 2009; 14: 251-7.

6. Targa L, Cardin G, Cozzi F, et al. Electrocardiographic disorders in diverse clinical variations of scleroderma. G Clin Med 1990; 71: 17-24.

7. Hata N, Kunimi T, Matsuda H, et al. Cardiac disorders associated with progressive systemic sclerosis. J Cardiol 1998; 32: 397-402. 
8. Roberts NK, Cabeen WR Jr, Moss J, et al. The prevalence of conduction defects and cardiac arrhythmias in progressive systemic sclerosis. Ann Intern Med 1981; 94: 38-40.

9. Bryan C, Knight C, Black CM, Silman AJ. Prediction of fiveyear survival following presentation with scleroderma: development of a simple model using three disease factors at first visit. Arthritis Rheum 1999; 42: 2660-5.

10. Van den Hoogen F, Khanna D, Fransen J, et al. 2013 classification criteria for systemic sclerosis: an American College of Rheumatology/European League against Rheumatism Collaborative Initiative. Ann Rheum Dis 2013; 72: 1747-55.

11. Khanna D, Furst DE, Clements PJ, et al. Standardization of the modified Rodnan skin score for use in clinical trials of systemic sclerosis. J Scleroderma Relat Disord 2017; 2: 11-8.

12. Dabrowski A, Piotrowicz R. Circadian rhythm of sinoatrial conduction time: a new approach to the study of sinoatrial node. CV World Report 1988; 1: 155-7.

13. Steen VD, Medsger TA Jr. Improvement in skin thickening in systemic sclerosis associated with improved survival. Arthritis Rheum 2001; 44: 2828-35.

14. Bienias P, Ciurzyński M, Korczak D, Pruszczyk P. Arrhythmias and conduction disturbances in patients with connective tissue diseases. Kardiol Pol 2008; 66: 194-9.

15. Huges M, Herrick AL. Raynauds phenomenon. Best Pract Res Clin Rheumatol 2016; 30: 112-32.

16. Allanore $Y$, Meune C. Primary myocardial involvement in systemic sclerosis: evidence for microvascular origin. Clin Exp Rheumatol 2010; 28 (Suppl. 62): 48-53.

17. Kramarz E, Piotrowicz R, Krzyżanowski K, Kramarz P. Comparison of sinoatrial conduction time measured by Holter metod and premature stimulation method. Ann Noninvasive Electrocardiol 2015; 20: 258-63.

18. Dąbrowski A, Kramarz E, Piotrowicz R. Prognostic significance of sinoatrial conduction time estimated using Holter ECG monitoring in patients with unexplained syncope. Kardiol Pol 1997; 46: 102-7.

19. Steinberg JS, Varma NV, Cygankiewicz I, et al. 2017 ISHEHRS expert consensus statement on ambulatory ECG and external cardiac monitoring/telemetry. Ann Noninvasive Electrocardiol 2017; 22: e12447.

20. Sobotka PA, Mayer JH, Bauernfeind RA, et al. Arrhythmias documented by 24-hour continuous ambulatory electrocardiographic monitoring in young woman without apparent heart disease. Am Heart J 1981; 101: 753-9.

21. Kramarz E, Makowski K. Clinical significance of second degree Wenckebach type sinoatrial block identified during Holter monitoring in patients with symptoms suggestive of arrhythmia. Europace 2015; 17: 123-30. 